\title{
Spectral Broadening of Gaussian Process in Optical Fibre and Implication on the Spectral Efficiency
}

\author{
Xianhe Yangzhang ${ }^{(1)(2)}$, Vahid Aref ${ }^{(2)}$, Domaniç Lavery ${ }^{(1)}$, Polina Bayvel(1) \\ (1) Optical Networks Group, UCL Department of Electronic \& Electrical Engineering, UK \\ (2) Nokia-Bell-Labs, Stuttgart, Germany
}

\begin{abstract}
We characterise the spectral evolution of Gaussian process in optical fibre using the Kolmogorov-Zakharov model. At high power regime, the spectrum at receiver is calculated by iteratively solving KZ-model at shorter distance. It questions the possibility of monotonically increasing spectral efficiency.
\end{abstract}

\section{Introduction}

The limit of the spectral efficiency (SE) of the optical fibre channel has been an unsolved problem in the optical communication society for decades. An upper bound ${ }^{4}$ on the capacity was derived for the point-to-point channel. Significant efforts have been made to search for a tighter upper bound or a non-saturating lower bound ${ }^{1,2,6,9}$. One related subtle problem is how to characterize the transmission bandwidth and in particular, the spectral broadening at at all range of powers.

In this work, this problem is approached from a trivial yet important aspect in a semi-analytical way - the spectral evolution of Gaussian process in optical fibre. The Kolmogorov-Zakharov (KZ) model is used to calculate the power spectral density (PSD) at the end of a link. At high power regime, the PSD at receiver is calculated by iteratively solving $\mathrm{KZ}$ model at a shorter distance. In-band energy ratio $\rho$ and $99 \%$-energy bandwidth are used to characterize the spectral broadening.

We show numerically that the in-band energy ratio can be approximately described by a monotonically decreasing function $\rho \approx f_{\rho}\left(\frac{P \sqrt{\mathcal{L}}}{W^{1.33}}\right)$. The result implies that: (i) Given a fixed processing bandwidth, increasing signal power of a Gaussian process causes more severe spectral broadening, limiting the achievable SE, (ii) if the signal bandwidth is increased while the power also grows proportional to $W^{(1+\epsilon)}$ for $\epsilon<$ 0.33 , the spectral broadening seems to be negligible. Hence, an ever-increasing spectral efficiency might become possible.

\section{The Optic-fibre Transmission Scenario}

The optic-fibre model of concern is a multi-span point-to-point dispersion unmanaged fibre link with equally-spaced erbium-doped fibre amplifier
Tab. 1: Fibre and system parameters

\begin{tabular}{|c|c|c|}
\hline$\nu$ & $193.44 \mathrm{THz}$ & center carrier freq. \\
$\alpha$ & $0.046 \mathrm{~km}^{-1}$ & fiber loss \\
$\gamma$ & $1.27(\mathrm{~W} \cdot \mathrm{km})^{-1}$ & nonlinearity para. \\
$\beta_{2}$ & $-21.5 \times 10^{-27} \mathrm{~s}^{2} / \mathrm{m}$ & dispersion para. \\
$W$ & $32 / 64 / 128 \mathrm{GHz}$ & linear bandwidth \\
$R_{o}$ & 4 & oversampling rate \\
$r$ & 0.01 & RRC roll-off factor \\
$\mathcal{L}_{\mathrm{sp}}$ & $80 \mathrm{~km}$ & span length \\
$N_{\mathrm{sp}}$ & $20 / 40 / 80$ & number of spans \\
\hline
\end{tabular}

(EDFA), which can be described by

$$
j \frac{\partial q}{\partial z}=-j \frac{\alpha}{2} q+\frac{\beta_{2}}{2} \frac{\partial^{2} q}{\partial t^{2}}-\gamma|q|^{2} q,
$$

where $j=\sqrt{-1}$ and $q(t, z)$ is the complex envelope of the signal as a function of time $t$ and distance $z$ along the fibre, $\beta_{2}, \gamma$, and $\alpha$ are the dispersion, non-linear, and loss coefficients of the fibre; see Tab. 1. Lumped amplification is performed at the end of the $k^{\text {th }}$ span with the power gain $g_{k}=\exp \left(\alpha \mathcal{L}_{\mathrm{sp}}\right)$, where $\mathcal{L}_{\mathrm{sp}}$ is the span length and $N_{\mathrm{sp}}$ is the number of spans.

Let assume the input signal $q(t, 0), 0<t<T_{0}$, to be a band-limited complex Gaussian (cyclo) stationary process, defined by its Fourier series

$$
q(t, 0)=\sum_{k=-\infty}^{\infty} Q_{k} \exp \left(j 2 \pi \frac{k}{T_{0}} t\right), \quad 0<t<T_{0}
$$

The Fourier coefficients $Q_{k}$ are independent symmetrically-complex Gaussian random variables with zero mean and the variance $S_{k}$. For a band-limited signal of bandwidth $W$, we have $S_{k}=0$ for $|k|>\left\lfloor W T_{0}\right\rfloor$. The power spectral density (PSD) of $q(t)$ is

$$
S_{q}(f)=S_{k} \delta\left(f-\frac{k}{T_{0}}\right) .
$$


Note that any desired power spectral density can be obtained through a suitable linear filter. Such an input signal satisfies the necessary conditions of the $\mathrm{KZ} \mathrm{model}{ }^{8}$ that $(i) Q_{k}$ are uncorrelated and, (ii) $q(t, z)$ has quasi-Gaussian distribution ${ }^{8}$ for all $z$ (under sufficiently weak nonlinearity framework). We further assume that the Fourier coefficients stays uncorrelated during the transmission.

Although the Gaussian process is a restrictive assumption, it has some practical relevance. In the current coherent optical transmission with relatively small launch power, the Gaussian-like input distribution, e.g. by probabilistic amplitude shaping, is of interest for the flexibility, high spectral efficiency as well as increasing the transmission reach. Moreover, the distribution of transmitted symbols converges anyhow to a quaziGaussian distribution after a long enough propagation distance and under sufficiently weak nonlinearity ${ }^{5,7}$.

\section{The Kolmogorov-Zakharov (KZ) Model}

We apply the KZ model for analytical characterisation of the spectral broadening. The $K Z$ model is a more suitable framework than the GN model ${ }^{5}$ simply because the $\mathrm{KZ}$ model is energypreserving ${ }^{8}$.

Let $\Delta z$ denote an integer multiple of span length $L_{\mathrm{sp}}$. We define $S_{k}[i]$ as the PSD of $q(t, z=$ $i \Delta z)$ after propagation of $i \Delta z / L_{\mathrm{sp}}$ spans. At the transmitter, $S_{k}[0]=S_{k}$. According to the $\mathrm{KZ}$ model $^{8}$, the PSD $S_{k}[i]$ can be recursively approximated as

$$
S_{k}[i+1] \approx S_{k}[i]+\underset{(l, m, n) \in V(k)}{\left.2 \gamma^{2} \sum_{l m n k}(\Delta z)\right|^{2} T_{l m n k}[i],}
$$

where

$$
\begin{aligned}
& V(k) \triangleq\{(l, m, n) \mid l+m=n+k, l \neq k, m \neq n\}, \\
& \Omega=j \alpha-\beta_{2}\left(\frac{2 \pi}{T_{0}}\right)^{2}\left(l^{2}+m^{2}-n^{2}-k^{2}\right), \\
& H_{l m n k}(\Delta z)=\frac{1-e^{j \Omega L_{\mathrm{sp}}}}{\Omega} e^{-j \Omega\left(\Delta z-L_{\mathrm{sp}}\right) / 2} \frac{\sin (\Delta z \Omega / 2)}{\sin \left(L_{\mathrm{sp}} \Omega / 2\right)}, \\
& T_{l m n k}[i]=S_{l}[i] S_{m}[i] S_{n}[i]+S_{l}[i] S_{m}[i] S_{k}[i] \\
& -S_{l}[i] S_{n}[i] S_{k}[i]-S_{m}[i] S_{n}[i] S_{k}[i] .
\end{aligned}
$$

Eq. (2) is only the first order perturbation approximation of the nonlinear kinetic equation Eq. (44) in $^{8}$. The accuracy of approximation depends on how small $\Delta z$ is chosen and depends on the power range. For relatively small power, one single iteration is quite accurate by $\Delta z=L_{\mathrm{sp}} \mathcal{N}_{\mathrm{sp}}$.
For a higher range of power, more iterations are required with smaller $\Delta z$ as illustrated in Fig. 1. The number of iterations, which varies with signal power and bandwidth, were empirically obtained in Fig. 1. Note that $\Delta z=L_{\mathrm{sp}}$ is the smallest $\Delta z$. We observed numerically that choosing smaller $\Delta z$ results more precise approximation than increasing the order of perturbation solution.

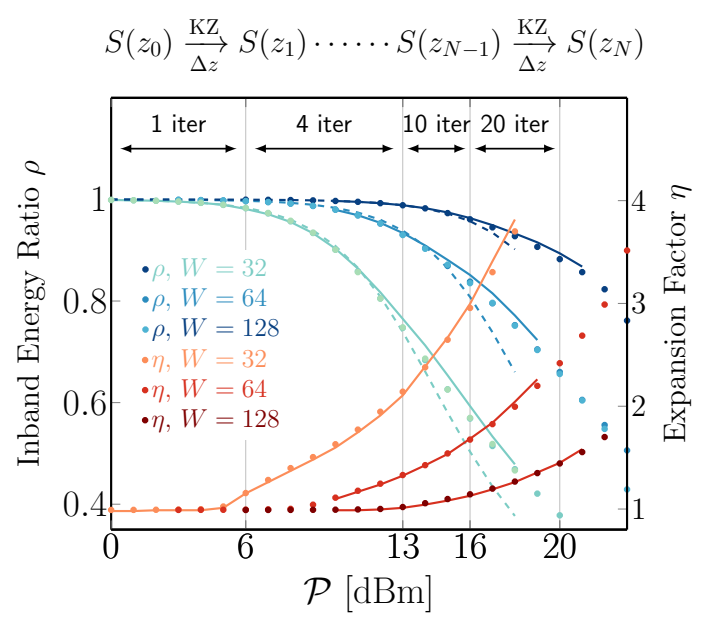

Fig. 1: Characterisation of spectral broadening in a 20-span transmission link with different initial bandwidths of 32/64/128 $\mathrm{GHz}$. Solid lines are results from KZ model, the number of iterations holds for $32 \mathrm{GHz}$ bandwidth. Dotted lines are from simulations with 16-QAM 0.01-RRC-pulse signals. Dashed lines are fitted curves.

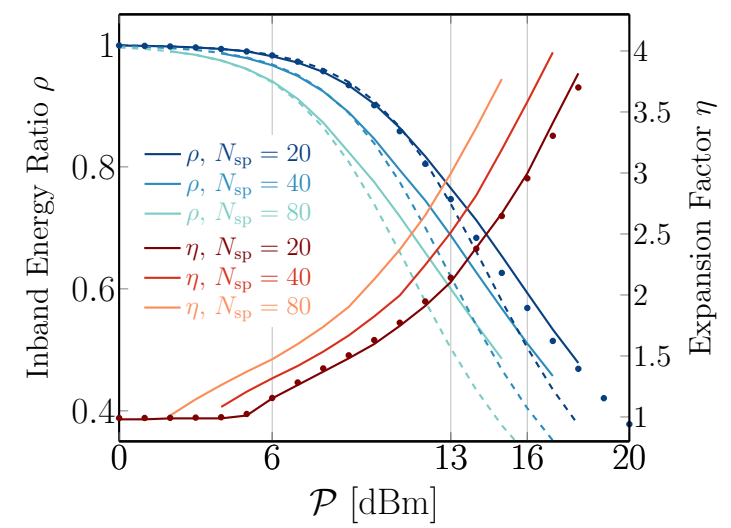

Fig. 2: Characterisation of spectral broadening of signals with $32 \mathrm{GHz}$ initial bandwidth at different transmission distances (20/40/80 spans). Solid lines are results from the KZ model, dots are from simulations. Dashed lines are fitted curves.

\section{Characterisation of Spectral Broadening}

We choose two figures of merit to characterise the spectral broadening:

(i) $\rho$ : the in-band energy ratio, the fraction of the energy preserved in the initial bandwidth $W$.

(ii) $\eta$ : the bandwidth expansion factor. The bandwidth is defined as the smallest frequency band holding more than $99 \%$ of energy.

We first compare the analytic estimation of (2) with the split-step Fourier simulation. The simulation parameters are outlined in Tab. 1. The 


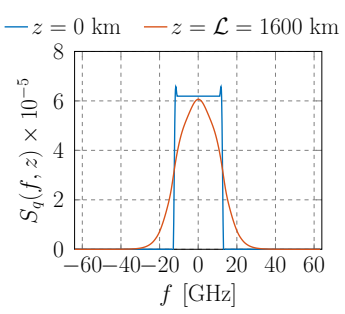

(a)

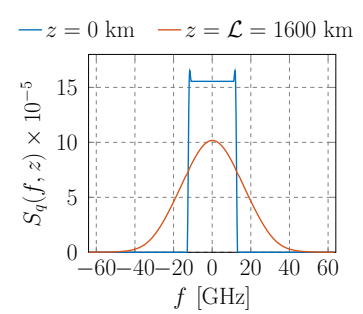

(b)
Fig. 3: Spectral broadening of a $32 \mathrm{GHz}$ signal at the power of (a) $12 \mathrm{dBm}$ and (b) $16 \mathrm{dBm}$.

amplifiers noise was skipped in the simulation. Figures 1 and 2 show the variation of $\rho$ and $\eta$ in terms of input launch power for different input signal bandwidth $W$ and for different number of spans. We observe that the analytic predictions (dashed lines) are quite consistent with the simulation results (dots). For not very large values of launch power $(\rho>0.9), \rho$ can be empirically approximated by,

$$
\rho(W, \mathcal{L}, \mathcal{P}) \approx \frac{1}{1+\ln \left(1+\zeta^{\mathcal{P}^{2} \mathcal{L}} W^{2.65}\right)},
$$

where $\mathcal{L}=L_{\mathrm{sp}} \mathcal{N}_{\mathrm{sp}}$ is the total distance, $\zeta$ is a constant, depending on the link parameters and the span length, $\mathcal{P}$ is the launch power in linear-scale (not in $\mathrm{dBm}$ ). We observe in Fig. 1 and 2 that the above approximation fits closely to the simulation for $\mathcal{P}<10 \mathrm{dBm}$. For larger values of $\mathcal{P}$, we observe that the scaling behaviour of $\rho(W, \mathcal{L}, \mathcal{P})$ and $\eta(W, \mathcal{L}, \mathcal{P})$ tends to become a function of $\frac{\mathcal{P} \sqrt{\mathcal{L}}}{W^{2}}$. It means that

$$
\rho \approx f_{\rho}\left(\frac{\mathcal{P} \sqrt{\mathcal{L}}}{W^{2}}\right), \quad \eta \approx f_{\eta}\left(\frac{\mathcal{P} \sqrt{\mathcal{L}}}{W^{2}}\right)
$$

\section{Discussion on the Spectral Efficiency}

It is shown recently ${ }^{4}$ that the spectral efficiency (SE) of the channel model (1), with ideal distributed amplification, is upper-bounded by the AWGN capacity

$$
\log \left(1+\frac{\mathcal{P}}{N_{\text {ase }} W_{\max }}\right)
$$

where $N_{\text {ase }}$ is the noise power spectral density and $W_{\max }$ is the maximum signal bandwidth. The recent result ${ }^{3}$ shows that, for dispersion-less fibre, for fixed initial bandwidth and large power, the bandwidth scales at least as a constant times $\sqrt{\mathcal{P}}$. For the channel with dispersion, we show a similar scaling in (4) for Gaussian input process.

Let us assume that, for EDFA-amplified links, the upper-bound (5) is still valid, then we have

$$
S E \leq \log \left(1+\frac{\mathcal{P}}{N_{\text {ase }} W f_{\eta}\left(\frac{\mathcal{P} \sqrt{\mathcal{L}}}{W^{1.33}}\right)}\right),
$$

If $\mathcal{P}$ and $W$ scale up at the same time with $\mathcal{P} \propto$ $\mathcal{W}^{(1+\epsilon)}$ where $\epsilon<0.33$, the $f_{\eta}$ will remain near 1 , i.e., the spectral broadening is negligible, while $\frac{P}{W}$ grows boundlessly.

Note that our scaling laws are derived for a Gaussian process and based on the result from iterative KZ-model without any ASE noise. Therefore, the scaling law can be different for an arbitrary input process in the highly nonlinear regime in which the $\mathrm{KZ}$ model is not valid any more.

\section{Conclusions}

In this work, we analysed the spectral evolution of Gaussian process with iterative KZ model solution for a large range of input powers. Conclusions are reached that 1) for a fixed bandwidth, the spectral efficiency of Gaussian process is limited by the effect of spectral broadening, 2) an ever-increasing SE might be possible if the signal bandwidth and power are increased at the same time with $\mathcal{P} \approx \mathcal{O}\left(W^{1.33}\right)$.

\section{Acknowledgements}

The work is within the COIN project, financed by the European Commission grant 676448, under the call H2020-MSCA-ITN-2015.

\section{References}

[1] E. Agrell et al. Influence of behavioral models on multiuser channel capacity. J. Lightw. Technol., 33(17):3507-3515, Sept 2015.

[2] V. Irukulapati et al. Stochastic digital backpropagation with residual memory compensation. J. Lightw. Technol., 34(2):566-572, Jan 2016.

[3] G. Kramer. Autocorrelation function for dispersionfree fiber channels with distributed amplification. arXiv:1705.00454, 2017.

[4] G. Kramer et al. Upper Bound on the Capacity of a Cascade of Nonlinear and Noisy Channels. arXiv:1503.07652, 2015.

[5] P. Poggiolini et al. A detailed analytical derivation of the gn model of non-linear interference in coherent optical transmission systems. arXiv:1209.0394, 2014.

[6] M. Secondini et al. Scope and limitations of the nonlinear shannon limit. J. Lightw. Technol., 35(4):893-902, Feb 2017.

[7] D. Soh et al. The effect of dispersion on spectral broadening of incoherent continuous-wave light in optical fibers. Opt. Express, 18(21):22393-22405, Oct 2010.

[8] M. I. Yousefi. The Kolmogorov-Zakharov Model for Optical Fiber Communication. IEEE Transactions on Information Theory, 63(1):377-391, Jan 2017.

[9] M. Yousefi et al. Linear and nonlinear frequency-division multiplexing. In Euro. Conf. on Opt. Communication (ECOC), Sept 2016. 